\title{
Synchrotron emission from the T Tauri binary system V773 Tauri A
}

\author{
M. Massi ${ }^{1}$, J. Forbrich ${ }^{1}$, K. M. Menten ${ }^{1}$, G. Torricelli-Ciamponi ${ }^{2}$, J. Neidhöfer ${ }^{1}$, S. Leurini ${ }^{1}$, and F. Bertoldi ${ }^{3}$ \\ 1 Max-Planck-Institut für Radioastronomie, Auf dem Hügel 69, 53121 Bonn, Germany \\ e-mail: mmassi@mpifr-bonn.mpg.de \\ 2 INAF, Osservatorio Astrofisico di Arcetri, Largo E. Fermi 5, 50125 Firenze, Italy \\ 3 Radioastronomisches Institut, Universität Bonn, Auf dem Hügel 71, 53121 Bonn, Germany \\ Received 30 May 2005 / Accepted 6 March 2006
}

\section{ABSTRACT}

\begin{abstract}
The pre-main sequence binary system V773 Tau A shows remarkable flaring activity around periastron passage. Here, we present the observation of such a flare at a wavelength of $3 \mathrm{~mm}(90 \mathrm{GHz})$ performed with the Plateau de Bure Interferometer ${ }^{\star}$. We examine different possible causes for the energy losses responsible for the e-folding time of $2.31 \pm 0.19 \mathrm{~h}$ of that flare. We exclude synchrotron, collisional, and inverse Compton losses because they are not consistent with observational constraints, and we propose that the fading of the emission is due to the leakage of electrons themselves at each reflection between the two mirror points of the magnetic structure partially trapping them. The magnetic structure compatible with both our leakage model and previous observations is that of a helmet streamer that, as in the solar case, can occur at the top of the X-ray-emitting, stellar-sized coronal loops of one of the stars. The streamer may extend up to $\sim 20 R_{*}$ and interact with the corona of the other star at periastron passage, causing recurring flares. The inferred magnetic field strength at the two mirror points of the helmet streamer is in the range $0.12-125 \mathrm{G}$, and the corresponding Lorentz factor, $\gamma$, of the partially trapped electrons is in the range $20<\gamma<632$. We therefore rule out that the emission could be of gyro-synchrotron nature: the derived high Lorentz factor proves that the nature of the emission at $90 \mathrm{GHz}$ from this pre-main binary system is synchrotron radiation.
\end{abstract}

Key words. stars: coronae - stars: individual: V773 Tau A - stars: flare - stars: pre-main sequence - radio continuum: stars stars: activity

\section{Introduction}

The dynamo theory (Parker 1955) explains how differential rotation generates a toroidal field in the interior of a star from an initial stellar dipole field, and how convection, bringing this field up to the surface, creates the coronal magnetic arc-like structures called loops. A close relationship between magnetic loops and flares exists. In fact, the physical mechanism invoked to explain solar and stellar flares today is magnetic reconnection, which occurs when different loops interact with each other or when field lines of the same loop are stretched. The released magnetic energy accelerates a part of the thermal electrons trapped in the loops to high energies, causing the flare (Golub \& Pasachoff 1997; Priest \& Forbes 2002).

We have observed a strong flare at $\mathrm{mm}$ wavelengths toward the T Tauri binary system V773 Tau A. In the context of the outlined scenario, our aim is to investigate: $a$ ) the flare location and hence the geometrical structure of the involved magnetic field; and $b$ ) the nature of the observed emission, i.e. whether the magnetic field intensity and the electron Lorentz factor can be derived, yielding information on the emission mechanism.

Concerning the first issue, $a$, one can distinguish among three possible origins for flares:

1. in a single star, flares occur when new emerging loops from below the stellar surface move into older, already-existing loops (Heyvaerts et al. 1977; Massi et al. 2005). Such intruding loops have indeed been observed on the Sun in

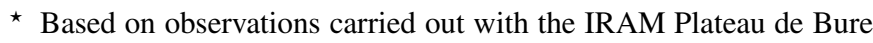
Interferometer. IRAM is supported by INSU/CNRS (France), MPG (Germany), and IGN (Spain). high-resolution maps obtained with the Nobeyama Radio Interferometer (Nishio et al. 1996);

2. in protostars or young (pre-main sequence) stellar systems, which are still surrounded by a dense accretion disk, flares can occur because of stretching, disruption, and reconnection of magnetic field lines between the star and its disk (Feigelson \& Montmerle 1999);

3. in close binary systems consisting of late spectral type stars, much higher magnetic activity is expected compared to the Sun (because tidal synchronism increases the rotation rate and therefore the dynamo efficiency). Moreover, the coronal loops of the two stars can interact with each other, as for RS CVn (close) binaries (Uchida \& Sakurai 1983; Graffagnino et al. 1995). Observational evidence for inter-binary loop "collisions" was also recently found for the young binary system V773 Tau A, which shows a high rate of radio flares around the periastron passage (Massi et al. 2002).

Concerning the second issue $(b)$, the usually observed radio emission from stellar coronae shows spectra peaking around $10 \mathrm{GHz}$ and is consistent with the gyro-synchrotron process (Mutel et al. 1985; Bastian et al. 1998; Güdel 2002). However, some solar and stellar flares can also be observed at millimeter wavelengths (Fig. 1), and the spectra are thought to be due to the superposition of a gyro-synchrotron spectrum with a synchrotron spectrum, the last one peaking in the shorter submillimeter to the far-infrared range (Kaufmann et al. 1988; Kaufmann et al. 2004). Gyro-synchrotron emission, associated with mildly relativistic particles, i.e., with a Lorentz factor of $\gamma<10$, is circularly polarized. In contrast, synchrotron emission comes from highly relativistic particles $(\gamma \gg 1)$ and is linearly polarized (Dulk 1985; Phillips et al. 1996; Tsuboi et al. 1998). 


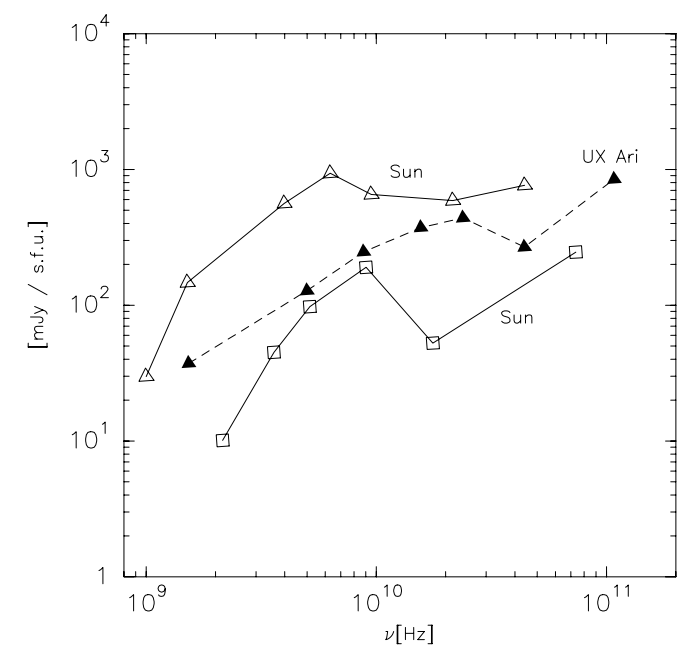

Fig. 1. Examples of spectra of the Sun (squares: Akabane et al. 1973; empty triangles: Zirin \& Tanaka 1973) and UX Arietis (filled triangles: Beasley \& Bastian 1998) exhibiting a "flattening" towards mmwavelengths. Notice that the vertical scale is in both solar flux units (for the solar spectra) and mJy (for UX Arietis).

To probe the existence of relativistic electrons in the V773 Tau A system and to constrain their energy and the strength and topology of the magnetic field, we observed it at millimeter wavelengths using the IRAM Plateau de Bure Interferometer.

The paper is organized as follows. Sect. 2 reviews previous results on V773 Tau A whereas in Sect. 3 our new observations and results are presented. In Sect. 4 we evaluate which kind of energetic processes may cause the trapped electrons to lose their energy and derive the Lorentz factor and magnetic field strength. These deductions are confirmed in Sect. 5 by the analysis of the flare rising time. Section 6 discusses the magnetic field configuration, while Sect. 7 presents our conclusions.

\section{The V773 Tau A binary system}

V773 Tau, at a distance of $148 \pm 5$ pc (Lestrade et al. 1999), is a quadruplet of $\mathrm{T}$ Tauri stars within an area of radius less than 100 AU (Woitas 2003). The system has two stars with orbital periods of years and two shorter period inner stars. The object of our studies is the V773 Tau A binary, with an orbital period of only 51.075 days (Welty 1995). The orbit of V773 Tau A is moderately eccentric $(e=0.3)$ and the epoch of periastron passage, corresponding to orbital phase $\Phi=0$, is $t_{0}=2449330.94 \mathrm{JD}$. The stars, both very active, have similar radii $\left(R_{*}=2.4 R_{\odot}\right)$ and rotation periods (about 3 days), but seem to have different masses (see Welty 1995).

The system possesses only weak hydrogen emission lines in its spectrum, which implies that its components are weak-line $\mathrm{T}$ Tauri stars with mass loss. This follows from the presence of forbidden emission lines (Cabrit et al. 1990). The presence of a disk is controversial (Skinner et al. 1997) since the observed infrared excess may also be attributable to V773 Tau D, the forth member of the quadruplet (Duchêne et al. 2003). Also, it is difficult to establish any thermal component in the millimeter emission (which would imply a disk), since it is strongly dominated by a variable non-thermal component typical of flaring coronal activity (Skinner et al. 1997). At $2.7 \mathrm{~mm}$, variability was found with the flux density changing from $30 \mathrm{mJy}$ to less than $3 \mathrm{mJy}$ in a period of a few months (see Skinner et al. 1997). Beckwith et al. (1990) measured a flux density at $1.3 \mathrm{~mm}$,
$S=42 \pm 6 \mathrm{mJy}$, whereas an observation several years later gave $S=24 \pm 4$ mJy (see Skinner et al. 1997). With the Effelsberg 100-m telescope, Massi et al. (2002) observed a flare at $7 \mathrm{~mm}$ with $S=68 \pm 13 \mathrm{mJy}$, and three months later they only obtained an upper limit $(3 \sigma)$ of $23 \mathrm{mJy}$.

V773 Tau A is not the only pre-main sequence object with mm-flaring emission. For example, Bower et al. (2003) observed a giant outburst at $86 \mathrm{GHz}$ in the Orion source GMR-A, also a weak-line $\mathrm{T}$ Tauri star. Long-lasting (13 days) millimeter activity in that source was observed by Furuya et al. (2003). However, V773 Tau A is the only known system with coronal solar-like magnetic activity where linearly polarized emission together with the more common circularly polarized emission was measured (Phillips et al. 1996). The high level of variable millimeter emission together with the presence of linear polarization indicates that a quite energetic electron population is present. The fact that these energetic electrons have rather short lifetimes is worthy of note for the following analysis. Phillips et al. (1996) find that total intensity variations at different wavelengths and epochs share common time scales and establish a recurring interval for the decay of radio events with a typical 1/e time scale of only about $1 \mathrm{~h}$.

The two components of V773 Tau A are both active (Welty 1995); flares on both of them are expected due to mechanism $a-1$ discussed in the introduction. In addition, however, there is evidence for mechanism $a-3$ (inter-binary loop collision): by folding the data, monitored over 522 days, with the orbital period (51.075 days), the flares appear to be clustered at periastron passage (Massi et al. 2002). Around the periastron, more than one interbinary interaction seems to occur: three consecutive flares, separated by a time interval of 3-4 days, have been observed during a continuous observation around periastron passage (Fig. 3 in Massi et al. 2002). The hypothesis of a stable active region simply eclipsed by the body of the star during each rotation ( $P_{\text {rot }}=3$ days) is ruled out by the short lifetimes (1-2 h only) of the energetic electrons (as discussed above and in Sect. 3). On the contrary, a large asymmetric structure rooted on one of the two rotating stars could explain the occurrence of the three consecutive flares, as repeated collisions (at each rotation) with the corona of the other star. The extent, $H$, of this magnetic structure must be large enough to allow the observed consecutive collisions around periastron passage. The periastron separation is 56 solar radii, $R_{\odot}$, which for a stellar radius, $R_{*}=2.4 R_{\odot}$ (Welty 1995), corresponds to $23 R_{*}$. Indeed, a large magnetic structure has been imaged by using Very Long Baseline Interferometry (VLBI, Phillips et al. 1996; orbital phase $\phi=0.1$ ) at $\lambda=3.6 \mathrm{~cm}$, with two peaks of different intensity separated by $0.17 \mathrm{AU}\left(15 R_{*}\right)$. Observational constraints for the size of the emitting region are therefore in the range $15-20 R_{*}$.

The fact that linear polarization has been observed implies that Faraday depolarization is not effective and puts an upper limit to the density $\left(n<10^{9} \mathrm{~cm}^{-3}\right)$ of the plasma confined in this extended magnetic structure (Phillips et al. 1996). X-ray observations give evidence not only of a density much above this limit, but also of the existence of smaller, solar-like, coronal loops in V773 Tau A. Skinner et al. (1997) interpreted the light curve of a hard X-ray flare in V773 Tau A as being due to the rotational modulation of the emitting flaring region, determining a size of $H \leq 0.6 R_{*}$. Tsuboi et al. (1998) interpreted the decay of another hard X-ray flare as being due to radiative cooling, obtaining a size of $1.4 R_{*}$. Therefore, radio and X-ray emission come from spatially separated regions: denser and smaller ones associated with X-ray emission and larger and more diffuse ones 


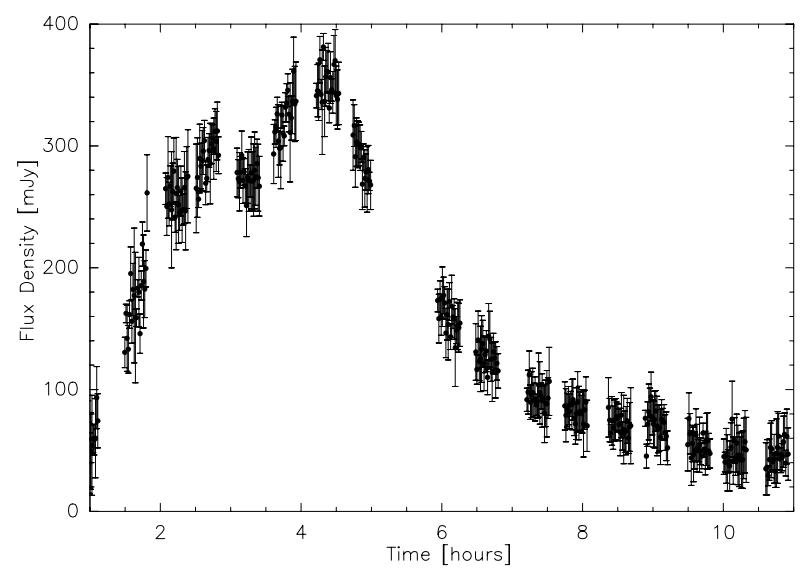

Fig. 2. V773 Tau A flare observed August 6, $2003(\Phi=0.1)$ with the Plateau de Bure Interferometer at $\lambda=3 \mathrm{~mm}$. The error bar corresponds to $2 \sigma$.

Table 1. Flux density in November 2003.

\begin{tabular}{lcc}
\hline \hline Day & $S_{1 \mathrm{~mm}}(\mathrm{mJy})$ & $S_{3 \mathrm{~mm}}(\mathrm{mJy})$ \\
\hline 13 & $5.0 \pm 1.2$ & $3.0 \pm 0.4$ \\
14 & $6.0 \pm 1.2$ & $5.0 \pm 0.3$ \\
\hline
\end{tabular}

associated with radio emission. Further confirmation of the presence of two structures comes from the multiwavelength campaign on V773 Tau A carried out by Feigelson et al. (1994) showing radio variability combined with a steady X-ray flux. Using our millimeter observations, the following sections are aimed to better define what kind of extended magnetic structure might confine the radio-emitting plasma, the intensity of the magnetic field, and the energy of the trapped particles.

\section{Observations and data reduction}

V773 Tau A was observed with the IRAM Plateau de Bure Interferometer at $1 \mathrm{~mm}$ and $3 \mathrm{~mm}$ around the two periastron passages in August and November 2003. The configuration was 5D (five antennas) in August and 6Cp (the six-antenna configuration) in November. Observations were done in LCP for the $3 \mathrm{~mm}$ observations and in RCP for the $1 \mathrm{~mm}$ observations. The continuum was measured with four correlator units per receiver, twice with a bandwidth of $320 \mathrm{MHz}$ and twice with a bandwidth of $160 \mathrm{MHz}$. Cyclical observations (every $20 \mathrm{~min}$ ) of the sources $0528+134$ and 3C286 were used for phase and amplitude calibrations. The data were reduced with the programs CLIC and MAPPING of the GILDAS software package developed by the Grenoble Astrophysics Group. The source was always unresolved. A complete flare (Fig. 2) was observed on August 6 $(\Phi=0.1)$. With about $12 \mathrm{~mm}$ of precipitable water vapor in the atmosphere, no observations at $\lambda=1 \mathrm{~mm}$ were possible, and those at $\lambda=3 \mathrm{~mm}$ had the high rms noise level of $17 \mathrm{mJy}$; the $3 \mathrm{~mm}$ receiver was tuned to $90 \mathrm{GHz}$ and had a system temperature around $1000 \mathrm{~K}$. The best power-law fit to the decay part of the light curve shown in Fig. 2 gives an e-folding time of $\tau=2.31 \pm 0.19 \mathrm{~h}$. Around the periastron passage in November, we were able to observe under very good weather conditions for two consecutive days at $\Phi=0$. The flux density was at a quiescent constant level with a nearly flat spectrum (Table 1).

\section{Rapid flare decay: leakage of the emitting particles}

As outlined in the introduction, the emission observed at $3.3 \mathrm{~mm}(90 \mathrm{GHz})$ can be interpreted as synchrotron radiation. In synchrotron radiation, the emission of each relativistic electron with Lorentz factor $\gamma$ moving in a magnetic field $B$ (in Gauss) is centered around a peak spectral frequency, $v_{0}$ (Ginzburg \& Syrovatski 1965) of

$v_{0}=1.8 \times 10^{6} B \gamma^{2} \mathrm{~Hz}$.

This implies that, to reproduce the observed emission at $v=$ $90 \mathrm{GHz}$, electrons must exist for which the following relationship holds:

$B \gamma^{2}=5 \times 10^{4}$.

The temporal evolution of the flux density shown in Fig. 2 clearly indicates that the emitting electron distribution, responsible for the observed synchrotron emission, is subject to some losses. In particular, the derived e-folding time of $2.31 \mathrm{~h}$ for the $\mathrm{mm}$ flare is of the same order of that measured by Phillips et al. (1996) for cm emission (about one hour). This time scale implies that the electrons must be at least partially trapped in the emitting region, which would otherwise be rapidly depleted in a time equal to the size of the region divided by the speed of the electrons: $20 R_{*} / c \sim 112 \mathrm{~s}$. It is well-established that particles trapped in magnetic structures spiral around field lines to a point (the "mirror point") where the magnetic field lines converge and the field strength is sufficient to cause the particles to reverse direction and travel back to the other mirror point. Reflected back and forth between mirror points (known as "bounce" motion), the particles remain trapped and, continuously spiraling, emit synchrotron radiation until they have lost their energy (Roederer 1970).

However, the decay time of $2.31 \mathrm{~h}$ is not attributable to synchrotron losses. As derived in the Appendix, synchrotron losses would explain an intensity decay time of that duration only for an emitting region of size $\leq 3.8 R_{*}$, whereas the observed size (Sect. 2) is $H \geq 15 R_{*}$. Neither inverse Compton losses, due to the radiation field of the star, and/or to the synchrotron emission of the flare (synchrotron self-Compton (SSC)), can be responsible for the observed decay; Compton losses are even lower than synchrotron losses (see appendix). On the other hand, even collisional losses cannot account for the observed decay time, since collisions are important only for high density plasmas (see appendix) in contrast to the upper limit on the plasma density $n<10^{9} \mathrm{~cm}^{-3}$ derived from Faraday depolarization (Sect. 2).

Since the fast decay of the emission cannot be attributed to energetic losses of the electrons, it may be caused by leakage of the particles themselves, meaning that at each bounce motion at one mirror point, some of the electrons are able to leave the trapping region and escape into free space. This is possible for electrons having a pitch angle, $\theta$ (the angle between their velocity direction and the magnetic field vector), smaller than the loss cone angle

$\sin \theta<\sin \theta_{0}=\left(\frac{B_{1}}{B_{0}}\right)^{1 / 2}$.

Here, $B_{1}$ is the magnetic field intensity far out in the corona at a distance $\left(H_{1}\right)$, while $B_{0}$ is that at a lower distance $\left(H_{0}\right)$. Pitch angle scattering, also known as "pitch angle diffusion" (Melrose \& Brown 1976), is one of the main processes by which trapped particles are lost. Weak and strong diffusion have been introduced by Melrose \& Brown (1976). However, in order to be effective, weak diffusion demands a high-density medium $\left(n>10^{9} \mathrm{~cm}^{-3}\right)$, in contrast to what we discussed above. Also, Lee et al. (2002) found that for the microwave decay of a solar flare, the assumption of strong diffusion seems to be the most appropriate. In this 
scenario, the emission decreases by a factor of $1 / \mathrm{e}$ if the precipitation rate

$v_{\mathrm{p}}=\frac{1}{2} \theta_{0}^{2} \frac{c}{\left(H_{1}-H_{0}\right)}$

(Melrose \& Brown 1976; Lee et al. 2002) times the decay time ( $2.31 \mathrm{~h}$, in our case) equals unity. This condition, valid for small $\theta_{0}$ values, implies:

$742 \times \frac{B_{1}}{B_{0}} \frac{R_{*}}{H_{1}-H_{0}}=1$.

For a magnetic dipole field, $B_{1} / B_{0}=\left[H_{0} / H_{1}\right]^{3}$, possible solutions of Eq. (5) are values in the ranges $H_{0} \sim(2-5) R_{*}$ and $H_{1} \sim(10-20) R_{*}$. Assuming a magnetic field strength at the stellar surface of the order of $1 \mathrm{kG}$ (see Appendix), the corresponding values of the magnetic field intensity are in the range 125-8 G for $H_{0}$ and $1-0.12 \mathrm{G}$ for $H_{1}$. This scenario is consistent with synchrotron emission, since from Eq. (2), the corresponding Lorentz factor of relativistic electrons emitting at $90 \mathrm{GHz}$ is $20<\gamma<632$.

This scenario is also in accordance with the VLBI observation of Phillips et al. (1996), since the analogon of Eq. (5) derived for the decay time of one hour and solved for $H_{1}-H_{0}=$ $15 R_{*}$ with $H_{1} \sim 20 R_{*}$ gives $B$ in the range of $4.6-0.12 \mathrm{G}$. These values of the magnetic field for $v=8.2 \mathrm{GHz}$ (Phillips et al. 1996) correspond (see Eq. (1)) to $31<\gamma<195$ indicating, therefore, the presence of relativistic electrons in agreement with the observed linear polarization.

\section{The flaring phase: a propagating shock}

In the previous section, we established the most likely physical process responsible for the rapid flare decay. The aim of this section is to test whether the additional constraint set by the flare rising time confirms the previously obtained results.

Recent solar flare studies (Tanuma \& Shibata 2005; Asai et al. 2004) have shown that the observed downflow motions can create fast shocks that are related to observed non-thermal bursts, since shocks are supposed to be sites of electron acceleration (Aschwanden 2002). In our scenario, in which reconnection takes place far out where the two stars' magnetospheres interact (i.e. at $H_{1}$ ), the shock induced by magnetic reconnection can propagate down to $H_{0}$ along the magnetic structure at the local Alfvén velocity

$v_{\mathrm{A}}(H)=\frac{B(H)}{\sqrt{4 \pi m_{H} n(H)}} \simeq 7 \times 10^{9}\left(\frac{H}{R_{*}}\right)^{-2} \mathrm{~cm} / \mathrm{s}$

(for a dipole magnetic field with $B\left(R_{*}\right)=1000 \mathrm{G}$ and a particle density $\left.n \sim 10^{9}\left(H / R_{*}\right)^{-2} \mathrm{~cm}^{-3}\right)$, thus inducing successive particle acceleration events. The shock propagation speed can be related to the flare rise time, $t_{\text {rise, }}$,

$t_{\text {rise }}=\int_{H_{0}}^{H_{1}} \frac{\mathrm{d} H}{v_{\mathrm{A}}(H)}$,

from which the following relationship can be derived:

$\left(\frac{H_{1}}{R_{*}}\right)^{3}=452\left(\frac{t_{\text {rise }}}{\mathrm{h}}\right)\left(\frac{B\left(R_{*}\right)}{10^{3} \mathrm{G}}\right)\left(\frac{n\left(R_{*}\right)}{10^{9} \mathrm{~cm}^{-3}}\right)^{-\frac{1}{2}}+\left(\frac{H_{0}}{R_{*}}\right)^{3}$.

For $t_{\text {rise }}, \simeq 4.5 \mathrm{~h}$ and $H_{0}$ between $(2-5) R_{*}$, the resulting height $H_{1}$ is in the interval of $(13-27) R_{*}$, assuming that $B\left(R_{*}\right)$ is in the range 1000-3000 $\mathrm{G}$ (see appendix) and the density is in
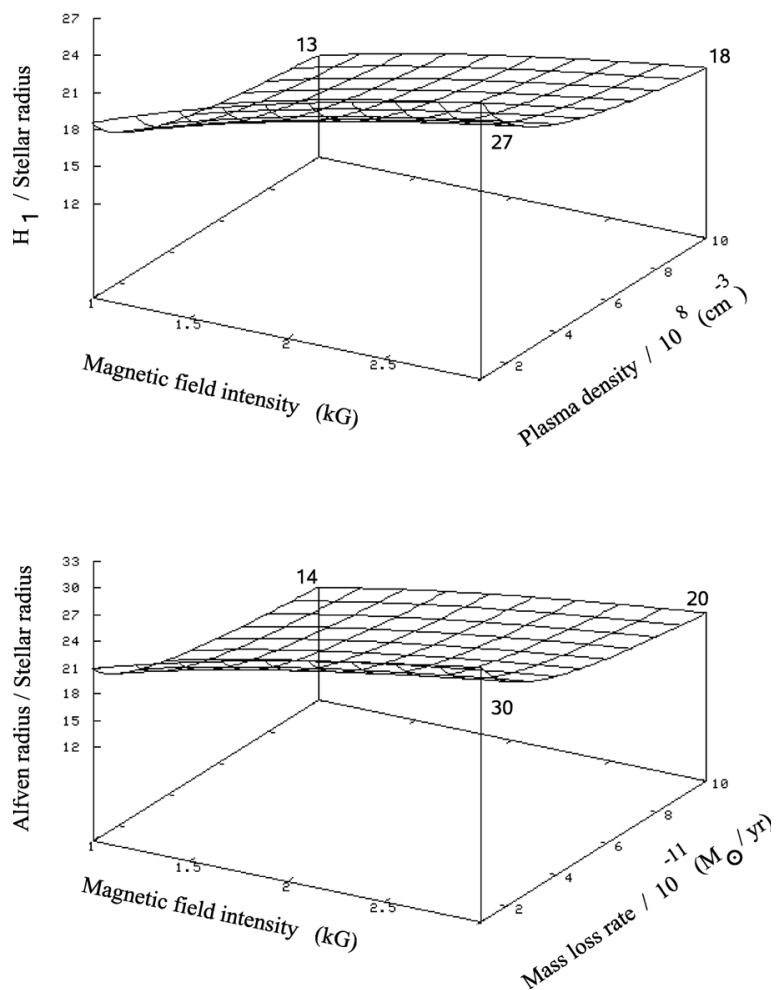

Fig. 3. Top: variations of the height $\mathrm{H}_{1}$ (Eq. (8)), where magnetic reconnection occurs, as a function of magnetic field intensity and plasma density (see Sect. 5). Bottom: Alfvén radius (Eq. (9)) as a function of magnetic field intensity and mass loss rate (see Sect. 6).

the range $10^{8}-10^{9} \mathrm{~cm}^{-3}$, as in Fig. 3. This range for $H_{1}$ is well-consistent with the scenario of a reconnection event at a large stellar distance and in agreement with the resulting interval (10-20) $R_{*}$ from the leakage model.

\section{Magnetic configuration}

In the previous sections, in the context of our leakage model, we established magnetic field values in the range 125-8 $\mathrm{G}$ (for $\left.H_{0}=2-5 R_{*}\right)$ and $0.12 \mathrm{G}\left(\right.$ at $H_{1}=20 R_{*}$ ) at the two mirror points of the confining magnetic structure. Also, we determined values of $4.6 \mathrm{G}$ (at $H_{0}=6 R_{*}$ ) and $0.12 \mathrm{G}$ (at $H_{1}=20 R_{*}$ ) for the centimeter observations of Philipps et al. (1996). In both cases, in a high-resolution image of the source brightness distribution, the two mirror points would correspond to two peaks (displaced by $H_{1}-H_{0}$ ) of rather different intensity, because of the relationship between emissivity and $B$ (Dulk 1985). This predicted morphology finds its confirmation in the Very Long Baseline Array (VLBA) image of Phillips et al. (1996). The VLBA image, given in the small box of Fig. 3, shows a strong brightness distribution peak, which we may identify with the first mirror point, $P_{0}$, separated by $15 R_{*}$ from a second weaker peak, which may be coincident with the second mirror point, $P_{1}$.

Let us assume the scenario of two stellar coronae interacting at periastron passage. One of the two coronae must be very asymmetric, in accordance with the asymmetric magnetic field configuration implied by the Massi et al. (2002) observations of three consecutive flares around periastron (see Sect. 2). On the other hand, a single giant stellar loop is ruled out by X-ray observations which, on the contrary (see Sect. 2), provide evidence for a stellar-sized coronal loop (Skinner et al. 1997; Tsuboi et al. 1998). However, in the case of the Sun, extended magnetic structures are sometimes present above coronal loops. 


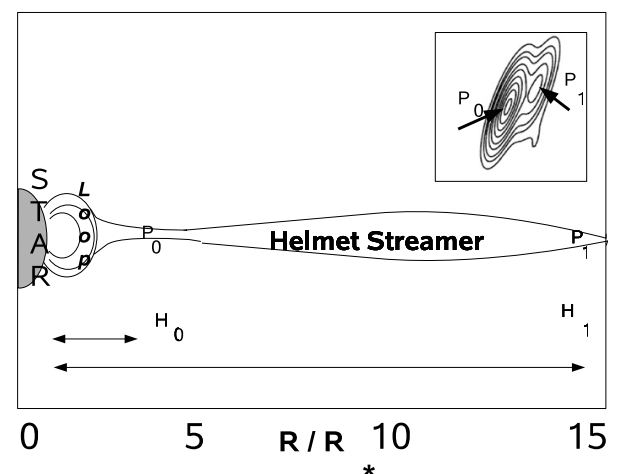

Fig. 4. Sketch of the magnetic structure of a helmet streamer, above a coronal loop, as treated in magnetohydrodynamic models (Endeve et al. 2004). The small box contains the VLBA image by Phillips et al. (1996).

Indeed, when the Sun is observed with white-light coronographs, one can see that above the top of some coronal loops (Fig. 4), a dome-shaped structure (the "helmet") is formed, extending out to 2-4 solar radii, with streamers that can extend out to many solar radii (Suess \& Nerney 2004; Endeve et al. 2004). A helmet streamer is therefore a plausible physical model for the elongated asymmetric structure implied by the previous observations of Massi et al. (2002). Moreover, a helmet streamer structure with its two mirror points at $P_{0}$ and $P_{1}$ (Fig. 4) matches the results of our leakage model based on PdBI data, as well as the appearence of the source in the VLBA image.

Finally, we point out that the helmet streamer in the simplified sketch of Fig. 4 is represented, for simplicity's sake, by a straight structure. In real highly conducting stellar plasmas, where the ionized gas is frozen to the field lines, more complex morphologies will occur: wherever magnetic pressure exceeds gas pressure, the fluid moves only along the field lines. In contrast, when gas pressure dominates, the magnetic field lines do move or bend, depending on the fluid motion. In a rotating star with mass loss rate $\dot{M}$ and terminal wind velocity $v_{\infty}$, the distance (called Alfvén radius, $R_{\mathrm{A}}$ ) where the corotation of the wind can no longer be enforced by the magnetic field and the magnetic field lines become curved is, as given by André et al. (1988),

$\frac{R_{\mathrm{A}}}{R_{*}}=26\left(\frac{B_{*}}{10^{4} \mathrm{G}}\right)^{\frac{1}{3}}\left(\frac{\dot{M}}{10^{-10} M_{\odot} \mathrm{yr}^{-1}}\right)^{-\frac{1}{6}}\left(\frac{v_{\infty}}{10^{3} \mathrm{~km} \mathrm{~s}^{-1}}\right)^{\frac{1}{6}}\left(\frac{P}{1 \mathrm{~d}}\right)^{\frac{1}{3}}$

Adopting a range $10^{-11}-10^{-10} M_{\odot} \mathrm{yr}^{-1}$ for the mass loss rate of a weak T Tauri star (André et al. 1992), and a wind terminal velocity of $300 \mathrm{~km} \mathrm{~s}^{-1}$ (Ardila et al. 2002), we can conclude that the Alfvén radius for $P=3 \mathrm{~d}$ (Fig. 3 bottom) is in the range of (14-30) $R_{*}$.

\section{Conclusions}

Our previous observations of the binary system V773 Tau A (Massi et al. 2002) have shown inter-binary loop collisions at periastron passage. We performed millimeter wavelength observations aimed to better define the extended magnetic structure confining the radio-emitting plasma, its topology, strength, and the energy of particles trapped in it. Based on our results, we propose the following scenario.

Radio and X-ray emission from V773 Tau A arise from spatially separated volumes: X-ray emission arises from denser and smaller regions (size $\lesssim 1 R_{*}$ ) like the closed loop region under $P_{0}$ in the sketch of Fig. 4 (see Sect. 2). The radio emission emerges from larger and more diffuse regions that are similar to the solar helmet streamers. A helmet streamer from one of the stars,

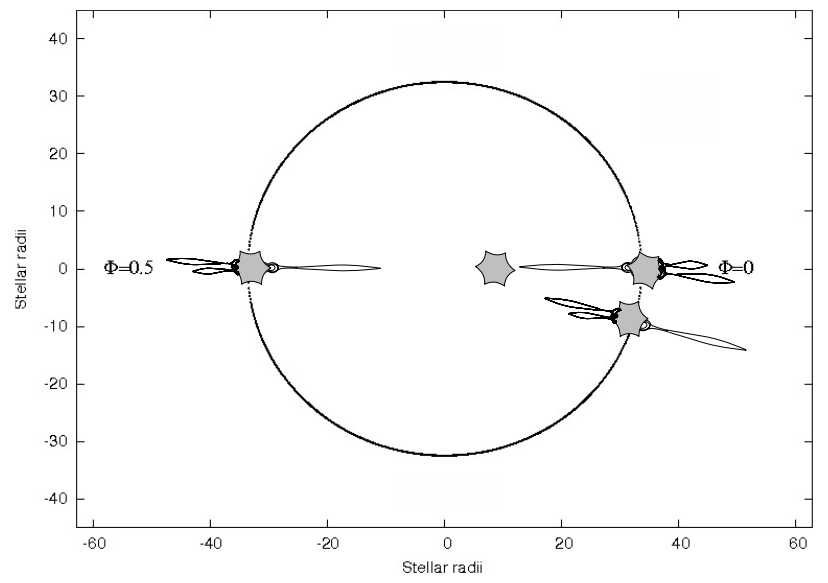

Fig. 5. Sketch of the binary system with the orbit parameters of Welty (1995). One star with helmet streamers is shown at different positions along the orbit. Interbinary collisions may occur at periastron passage (see text).

formed at the top of a coronal loop, as in the case of the Sun, could interact with the corona of the other star and could produce the strong radio flares observed around periastron passage (Massi et al. 2002). In this scenario, the lack of flares at some periastron passage (as in the PdBI observations of Table 1) could be explained as the absence of a collision, possibly because no large enough helmet streamer was emerging on the hemisphere facing the other star. As shown in Fig. 5, because of the slightly eccentric orbit $(e \simeq 0.3$, Welty 1995) the distance at periastron (23 $\left.R_{*}\right)$ is appreciably less than at apoastron $\left(40 R_{*}\right)$. The size of the helmet streamer should therefore be comparable with the periastron distance, but still be less than $40 R_{*}$. In this paper, where we report a flare at $3 \mathrm{~mm}$ with $S_{\text {peak }}=360 \pm 17 \mathrm{mJy}$, we have derived the size " $H_{1}$ " of the emitting region (i.e. the helmet streamer) in three completely independent ways. Interpreting the rising time of the flare in terms of propagation towards the low corona of a shock due to a magnetic reconnection event occurring at large height, we have determined a height $H_{1}$ in the range of $(13-27) R_{*}$. This size agrees with that determined for the Alfvén radius, i.e. in the range (14-30) $R_{*}$. By interpreting the rapid decay of the flare as leakage of the emitting electrons from the trapping magnetic structure of size $H_{1}$, we determine $H_{1}$ in the range (10-20) $R_{*}$. As a result, one of the two stars of the system might have a corona of a few stellar radii only, and interbinary collisions can still occur because of a large (i.e. average size $H_{1} \sim 19 R_{*}$ ) helmet streamer located on the other star.

The leakage model has shed light on the nature of the electrons responsible for the flare. We state that interbinary collisions are able to accelerate electrons to relativistic velocities. In fact, we determine a Lorentz factor $\gamma$ up to 632. This confirms that the emission in this pre-main sequence system is synchrotron radiation, as postulated from the observed linear polarization (Phillips et al. 1996).

In particular, streaming back and forth between the two mirror points $\left(P_{0}\right.$ and $P_{1}$ in Fig. 4$)$ of the helmet streamer, with magnetic fields between $0.12 \mathrm{G}$ and $125 \mathrm{G}$, the relativistic electrons produce the observed synchrotron radiation. The trapping by the helmet streamer is, however, only partial and during each reflection particles escape and the emission fades out relatively quickly. Leakage explains the puzzling finding that flares have similar decay times ( $1-2 \mathrm{~h}$ ), not only in different radio bands, but also in the millimeter band. The latter similarity would be difficult to explain on the basis of any loss mechanism dependent on the electron energy. 
Acknowledgements. We thank the referee for reading the manuscript with great attention and providing many constructive comments and critical remarks. We acknowledge the IRAM staff from the Plateau de Bure and from Grenoble, in particular Jan Martin Winters, for their help provided during the observations and data reduction. IRAM is an international institute for research in millimeter astronomy funded by the Max Planck Gesellschaft, Germany, the Centre National de la Recherche Scientifique, France, and the Instituto Geografico Nacional, Spain.

\section{Appendix A: Energetic considerations}

Accelerated non-thermal electrons are subject to energy losses both because synchrotron radiation is emitted and because of collisions with thermal electrons. In addition, energy losses are also due to the interaction of non-thermal electrons by inverse Compton (IC) processes with the stellar radiation field (external IC) and even with photons emitted by the synchrotron process itself in the radio band (synchrotron self-Compton (SSC)).

If synchrotron losses are responsible for the observed decay ( $\tau$, in hours), the following relationship holds between magnetic field intensity $B$ (in Gauss) and Lorentz factor $\gamma$ (Blumenthal \& Gould 1970):

$\gamma B^{2}=\frac{2.2 \times 10^{5}}{\tau_{\text {synchrotron }}}$.

From Eqs. (A.1) and (2), with $\tau_{\text {synchrotron }}=2.31 \mathrm{~h}$, the value of the magnetic field strength in the emitting region and the value of the Lorentz factor of the emitting electrons can be derived as: $B=56 \mathrm{G}$ and $\gamma=30$.

A magnetic dipole field, with $B\left(R_{*}\right)$ at the stellar surface, attains the intensity of $56 \mathrm{G}$ at a distance $H / R_{*}=\left[B\left(R_{*}\right) / 56\right]^{\frac{1}{3}}$. In the literature, there are very few estimates of the magnetic flux $B\left(R_{*}\right)$ in weak T Tauri stars. Basri et al. (1992) determined the upper limit of $1500 \mathrm{G}$ for the product $B f$, where $f<1$, for TAP 35. An even higher value $(\mathrm{B} f>2000 \mathrm{G})$ is derived by Guenther et al. (1999) in the source LkCa 16. Basri et al. (1992) exclude fields above $3000 \mathrm{G}$, and more recently (Bower et al. 2003), the value of $B=2600 \mathrm{G}$ has been measured in a weak $\mathrm{T}$ Tauri in the Orion Nebula. Therefore, 1000-3000 G seems to be a likely range. For $B\left(R_{*}\right) \leq 3000 \mathrm{G}$, the magnetic dipole field attains the intensity of $56 \mathrm{G}$ at a distance $H / R_{*} \leq 3.8$ from the stellar surface. This scenario cannot account for the observed extended radio emission (Sect. 2). We also exclude synchrotron losses as being responsible for the lifetime $\tau=1 \mathrm{~h}$ of the Phillips et al. (1996) observations at $v_{0}=8.2 \mathrm{GHz}$. The application of Eqs. (1) and (A.1) would give $B \sim 223 \mathrm{G}$ and $\gamma=4$, and hence emission coming from a region relatively close to the stellar surface $\left(H / R_{*} \leq 2.4\right)$ and from mildly relativistic electrons, in contrast with previously reported considerations (Sects. 1 and 2).

IC losses cannot account for the observed emission decay either. In fact, as derived hereafter, IC losses are even lower than synchrotron losses because the radiation energy density $(U)$ is smaller than the magnetic energy density i.e.,

$U<B^{2} / 8 \pi$.

A dipole magnetic field, with the intensity at the surface of the star in the range quoted above (say $2 \times 10^{3} \mathrm{G}$ ), has $B^{2} / 8 \pi \simeq 1.6 \times 10^{5}\left(H / R_{*}\right)^{-6} \mathrm{erg} \mathrm{cm}^{-3}$, while $U=L_{*} / 4 \pi c H^{2}=$ $0.86\left(H / R_{*}\right)^{-2} \mathrm{erg} \mathrm{cm}^{-3}$ for known values of radius and luminosity of V773 Tau A $\left(R_{*} \sim 2.4 R_{\odot}, L_{*} \sim 3 L_{\odot}\right)$ (Skinner et al. 1997; Welty 1995). Hence, if the radiation of the star is the source of the seed photons (IC), condition (A.2) holds for $H \leq 21 R_{*}$. On the other hand, for SSC, the related flare luminosity is $L_{\text {flare }}=6.4 \times 10^{26} \mathrm{erg} \mathrm{s}^{-1}$, which, supposing that the flare takes place in a spherical region of radius $a R_{*}$, with $a>0.01$, gives $U=6 \times 10^{-8} a^{-2} \mathrm{erg} \mathrm{cm}^{-3}$. Hence, condition (A.2) holds throughout the region of interest, since $\left(H / R_{*}\right) \leq 118 a^{1 / 3}$ is verified for every $\left(H / R_{*}\right) \leq 25$.

Finally, the thermalization time due to collisions of relativistic electrons in an ionized gas of density $n$ is given by (Petrosian 1985; Massi \& Chiuderi-Drago 1992):

$\tau_{\text {collisions }} \simeq 4.16 \times 10^{8} \frac{\gamma}{n}$

where $n$ is in $\mathrm{cm}^{-3}$ and $\tau$ is expressed in hours. Therefore, collisions would be important only for $n \sim \gamma 1.8 \times 10^{8} \mathrm{~cm}^{-3}$, which, for the relativistic electrons $(\gamma \gg 1)$ implied by the linear polarization, does not match the condition derived from Faraday depolarization $\left(n<1 . \times 10^{9} \mathrm{~cm}^{-3}\right.$; see Sect. 2$)$.

\section{References}

André, P., Montmerle, T., Feigelson, E. D., Stine, P. C., \& Klein, K. 1988, ApJ, 335, 940

André, P., Deeney, B. D., Phillips, R. B., \& Lestrade, J. 1992, ApJ, 401, 667 Akabane, K., Nakajima, H., Ohki, K., Moriyama, F., \& Miyaji, T. 1973, Sol. Phys., 33, 431

Ardila, D. R., Basri, G., Walter, F. M., Valenti, J. A., \& Johns-Krull, C. M. 2002, ApJ, 567, 1013

Asai, A., YokoYama, T., Shimojo, M., \& Shibata, K. 2004, ApJ, 605, L80

Aschwanden, M. J. 2002, Space Sci. Rev., 101, 1

Basri, G., Marcy, G. W., \& Valenti, J. A. 1992, ApJ, 390, 662

Bastian, T. S., Benz, A. O., \& Gary, D. E. 1998, ARA\&A, 36, 131

Beasley, A. J., \& Bastian, T. S. 1998, Radio Emission from Galactic and Extragalactic Compact Sources, ed. J. A. Zensus, G. B. Taylor, \& J. M. Wrobel, ASP Conf. Ser., 144, 321

Beckwith, S. V. W., Sargent, A. I., Chini, R. S., \& Guesten, R. 1990, AJ, 99, 924 Bower, G. C., Plambeck, R. L., Bolatto, A., et al. 2003, ApJ, 598, 1140 Blumenthal, G. R., \& Gould, R. J. 1970, Rev. Mod. Phys., 42, 237 Cabrit, S., Edwards, S., Strom, S. E., \& Strom, K. M. 1990, ApJ, 354, 687 Duchêne, G., Ghez, A. M., McCabe, C., \& Weinberger, A. J. 2003, ApJ, 592, 288 Dulk, G. A. 1985, ARA\&A, 23, 169

Endeve, E., Holzer, T. E., \& Leer, E. 2004, ApJ, 603, 307

Feigelson, E. D., \& Montmerle, T. 1999, ARA\&A, 37, 363

Feigelson, E. D., Welty, A. D., Imhoff, C., et al. 1994, ApJ, 432, 373

Furuya, R. S., Shinnaga, H., Nakanishi, K., Momose, M., \& Saito, M. 2003, PASJ, 55, L83

Ginzburg, V. L., \& Syrovatski, S. I. 1965 ARA\&A, 3, 297

Golub, L., \& Pasachoff, J. M. 1997, The Solar Corona (Cambridge: Cambridge University Press), 284

Graffagnino, V. G., Wonnacott, D., \& Schaeidt, S. 1995, MNRAS, 275, 129

Güdel, M. 2002, ARA\&A, 40, 217

Guenther, E. W., Lehmann, H., Emerson, J. P., \& Staude, J. 1999, A\&A, 341, 768 Heyvaerts, J., Priest, E., \& Rust, D. M. 1977, Sol. Phys., 53, 255

Kaufmann, P., Correia, E., Costa, J. E. R., \& Zodi Vaz, A. M. 1986, A\&A, 157, 11

Kaufmann, P., Raulin, J. P., de Castro, C. G., et al. 2004, ApJ, 603, 121

Lee, J., Gary, D. E., Qiu, J., \& Gallagher, P. T. 2002, ApJ, 572, 609

Massi, M., \& Chiuderi-Drago, F. 1992, A\&A, 253, 403

Massi, M., Menten, K., \& Neidhöfer, J. 2002, A\&A, 382, 152

Massi, M., Neidhöfer, J., Carpentier, Y., \& Ros, E. 2005, A\&A, 435, L1

Melrose, D. B., \& Brown, J. C. 1976, MNRAS, 176, 15

Mutel, R. L., Lestrade, J. F., Preston, R. A., \& Phillips, R. B. 1985, ApJ, 289, 262

Nishio M., Yaji K., \& Kosugi T. 1996, Radio Emission from the Stars and the Sun, ed. A. R. Taylor, \& J. M. Paredes, ASP Conf. Ser., 93, 378

van den Oord, G. H. J., Mewe, R., \& Brinkman, A. C. 1988, A\&A, 205, 181

Parker, E. N. 1955, ApJ, 121, 49

Phillips, R. B., Lonsdale, C. J., Feigelson, E. D., \& Deeney, B. D. 1996, AJ, 111, 918

Priest, E. R., \& Forbes, T. G. 2002, A\&AR, 10, 313

Roederer, J. G. 1970, Dynamics of Geomagnetically Trapped Radiation (Berlin: Springer-Verlag)

Skinner, S. L., Guedel, M., Koyama, K., \& Yamauchi, S. 1997, ApJ, 486, 886

Suess, S. T., \& Nerney, S. 2004, Advances in Space Research 33, 668

Tanuma, S., \& Shibata, K. 2005, ApJ, 628, L77

Tsuboi, Y., Koyama, K., Murakami, H., et al. 1998, ApJ, 503, 894

Uchida, Y., \& Sakurai, T. 1983, IAU Coll., 71, 629

Welty, A. D. 1995, AJ, 110, 776

Woitas, J. 2003, A\&A, 406, 685

Zirin, H., \& Tanaka, K. 1973, Sol. Phys., 32, 173 